\title{
Skeletal Muscle Depletion and Nutrition Support Affected Postoperative Complications in Patients Underwent Pancreatoduodenectomy
}

\section{Kaipeng Duan}

The First Affiliated Hospital of Soochow University

\section{Xin Gao}

The First Affiliated Hospital of Soochow University

\section{Luxin Wei}

The First Affiliated Hospital of Soochow University

\section{Mengting Gong}

The First Affiliated Hospital of Soochow University

\section{Jin Zhou}

The First Affiliated Hospital of Soochow University

\section{Dongming Zhu ( $\nabla$ zdmsdfyy@126.com )}

The First Affiliated Hospital of Soochow University

\section{Research}

Keywords: Skeletal muscle depletion, pancreatoduodenectomy, complications, nutrition therapy

Posted Date: August 25th, 2020

DOI: https://doi.org/10.21203/rs.3.rs-60117/v1

License: (1) This work is licensed under a Creative Commons Attribution 4.0 International License. Read Full License 


\section{Abstract}

\section{Background}

Postoperative complications remain high in patients underwent pancreaticoduodenectomy (PD). Body composition has been shown closely related to the outcome in critical and surgical patients. The aim of the present study was to investigate whether preoperative skeletal muscle condition and postoperative nutrition would affect major complications in patients underwent PD.

\section{Methods}

This retrospective study included 265 patients underwent PD with a CT scan of the abdomen within 1 week before surgery. Body compartment data was extracted from the L3 level of the CT scan, which included skeletal muscle area and density, subcutaneous and visceral fat area. Univariable and multivariable regression analyses were performed to investigate correlations between the above variables and postoperative complications. Furthermore, a subgroup analysis was conducted to explore the relationship between postoperative nutrition strategy and the outcome.

\section{Results}

Of all the 265 patients, the mean age and BMI was $59.5 \pm 13.9$ years and $23.1 \pm 3.6 \mathrm{~kg} / \mathrm{m}^{2}$, respectively. Major complications occurred in 81 patients (30.6\%). Cutoff values for skeletal muscle depletion were defined by ROC curve analysis from postoperative complications in skeletal muscle index (SMI) (male $47.32 \mathrm{~cm}^{2} / \mathrm{m}^{2}$ and female $40.65 \mathrm{~cm}^{2} / \mathrm{m}^{2}$ ). Univariable analysis showed postoperative complications were significantly associated with age, sex, systemic comorbidity, SMI, skeletal muscle density (SMD) and visceral adipose tissue index (VATI). Multivariable regression reveled only age (OR 1.49, 95\% $\mathrm{Cl} 1.22-$ 1.83, $\mathrm{p}=0.026)$, SMI (OR 0.77, 95\% Cl 0.51-0.94, $\mathrm{p}=0.015)$ and SMD (OR 0.85, 95\% $\mathrm{Cl} 0.64-1.03, \mathrm{p}=0.029)$ were independent predictors for major complications. Subgroup analysis showed the initial parenteral nutrition time (IPNT) (OR1.89, 95\% Cl 1.43-2.49, p=0.032) and average protein delivery (APD) (OR 0.76, $95 \% \mathrm{Cl} 0.53-0.89, \mathrm{p}=0.021$ ) were significantly associated with major complications in patients with lower SMI. However, in normal SMI patients, such association was not significant enough.

\section{Conclusions}

Preoperative skeletal muscle index and density were independently associated with major complications in patients underwent PD. In patients with lower SMI, early parenteral nutrition and higher protein delivery were related to better outcome.

\section{Introduction}

Pancreaticoduodenectomy (PD) is an important surgical procedure for malignant and benign disease of pancreatic head and periampullary region. With the development of surgery and critical care, the mortality has dropped significantly in the past decades. However, the rate of postoperative complications remains 
high and hinders the recovery and long-term outcome. Although, several technical strategies have been proposed to reduce perioperative complications, their availability is still controversial. Therefore, assessment and identification of the high-risk patients have progressively gained attention.

Recently, the loss of skeletal muscle, or sarcopenia, was recognized as an independent factor affecting the prognosis in cancer patient [1]. Similarly, critical patients with more depletion of muscle mass tend to have longer ventilation and lower survival $[2,3]$. Patients undergoing major surgery, such as PD, constituted a large portion of intensive care unit (ICU) patients, and received more attention of research for the comprehensive premorbid data. Lately, preoperative visceral obesity and sarcopenia have been shown associated with major complications in patients with pancreatic ductal adenocarcinoma (PDAC) $[4,5]$. Meanwhile, several studies began to reveal the close relationship between sarcopenia and postoperative pancreatic fistula (POPF) in patients receiving PD [6-8]. However, the definition and diagnosis of sarcopenia varied greatly in these studies and body composition differed in various population $[9,10]$. So it is necessary to validate the predictive value of skeletal muscle depletion on postoperative complications in PD from a large cohort population.

Patients receiving PD are always admitted to ICU electively or in response to complications. In critical patients after major upper gastrointestinal surgery where early enteral nutrition (EN) is limited, the timing and caloric/protein target of parenteral nutrition $(\mathrm{PN})$ remain to be a controversial issue $[11,12]$. Data from a meta-analysis showed a significant decrease of infectious morbidity with preoperative and early postoperative PN compared with standard therapy (STD) [13]. However, the beneficial effect appeared vanished if PN given only postoperatively and there could be even worse outcome with early nutrition [13]. A recent randomized control trial (RCT) demonstrated no difference in 60-day mortality whether critical patients ( $>60 \%$ surgical admission) received early PN or STD [14]. These discrepancies may result from the heterogeneous critical patients with different baseline nutritional conditions or diseases. Routine nutrition risk assessment, such as NRS-2002 and NUTRIC may have limited power to identify those surgical patients requiring imperative nutrition support, since they mainly focus on weight alteration or general conditions. Given the pivotal role of skeletal muscle in nutritional assessment, we hypothesized skeletal muscle depletion might impact the efficiency of nutrition therapy in patients undergoing PD. Therefore, this study aimed to evaluate the effects of preoperative skeletal muscle condition and postoperative PN on major complications in patients underwent PD procedure.

\section{Methods}

\section{Patients and data}

This is a retrospective analysis of surgical patients underwent PD from January 2014 to December 2019 in a tertiary hospital (The First Affiliated Hospital of Soochow University). Patients were included if they were aged 18 years or older, underwent PD procedure, and had an preoperative abdominal CT scan made no more than 1 week before surgery. The excluded criteria were with preoperative organ failure, poor quality CT unable to analyze, and incomplete medical records. 
Patient data included age, sex, weight, height, admission diagnosis, body mass index (BMI), NRS2002 scores and routine preoperative blood test. The primary outcome parameter of this research was major postoperative complications of Clavien-Dindo Classification (CDC) $\geq 3$ [15]. Among these complications $(C D C \geq 3)$, POPF was graded according to the International Study Group for Pancreatic Fistula classification [16], and nosocomial infection included confirmed incision infection, abdominal infection and lung infection, which need specific treatment, such as upgrading antibiotics, secondary closure and additional drainage. Second outcome included length of hospitalization, the postoperative time to discharge, and 28-day mortality. The average calories delivery (ACD) and average protein delivery (APD) were calculated from the total calories and protein patients received both EN and PN in the first week after surgery. The initial parenteral nutrition time (IPNT) was defined as the time interval from the end of the surgery to the beginning of PN in hours.

\section{Surgical procedure and perioperative management}

All patients received Whipple or pylorus-preserving PD procedure conducted by experienced pancreatic surgeons. A two-layer duct to mucosa pancreato-jejunostomy with either Child or Roux-en-Y technique was used for reconstruction with at least two surgical drains in the abdomen, one next to the bilio-jejunal anastomosis and one closed to the pancreato-jejunal anastomosis. Somatostatin or its analogue was routinely administered for three to seven days. Enteral nutrition was initiated as early as possible and parenteral nutrition was used as a supplementary part according to the patients' condition. Other management included proton pump inhibitor, antibiotics, anti-coagulation and encouraged early mobilization.

\section{CT scan analysis}

The pre-operative abdominal CT test was performed no more than 1 week before surgery. Abdominal CT scans were analyzed in anonymized format by two blinded independent researcher trained in radiologic anatomy and body composition analysis. Briefly, a single slice of unenhanced CT scan with a 5-mm thickness at the third lumbar vertebra (L3) level was extracted from picture archiving and communication system (PACS), since both skeletal muscle area $(r=0.83-0.99 ; p<0.01)$ and intermuscular adipose tissue (IMAT) $(r=0.39-0.61 ; p<0.05)$ at this level are closely related to whole body skeletal muscle and IMAT volumes [17]. Skeletal muscles included into the analysis were the internal and external obliques, transversus abdominus, rectus abdominus, psoas, quadratus lumborum, and erector spinae muscles. Tissue attenuation ranging from -29 to +150 Hounsfield Units (HU) was identified as skeletal muscle, 190 to - 30 for IMAT and subcutaneous adipose tissue, and - 150 to - 50 for visceral adipose tissue [18]. The tissue area and density was measured by ImageJ version 1.8.0 (National Institutes of Health). The skeletal muscle index (SMI) was calculated from L3 muscle area $(\mathrm{cm})$ divided by height square $\left(\mathrm{m}^{2}\right)$.

\section{Statistics}

Categorical variables were compared by Fisher Exact- and $\chi 2$ tests. Continuous variables with a normal distribution were compared by $t$ test, and Mann-Whitney $U$ test for those non-normally distributed. 
Receiver operating characteristic (ROC) curve analysis was used to define the cutoff values of body composition best fit to predict complications $(C D C \geq 3)$ in female and male patients separately.

Logistic regression analysis was used to evaluate the association between body composition data, nutrition therapy and major complication. After univariable analysis, a multivariable analysis was performed adjusted for sex, age, and BMI. Any variable that generated a P-value of $<0.1$ in univariable analysis was added to the multivariable analysis. Then the predicted probability of the final multivariable model for complication was assessed by ROC curves. Furthermore, we conducted a subgroup logistic regression analysis to evaluate the effect of nutrition therapy on postoperative complications in patients with low and normal SMI respectively. Restricted cubic splines were fit by a logistic regression model with the mean value as the reference standard. In both univariable and multivariable analysis, body composition data was used as categorical variables, with the thresholds from former ROC analysis, and the other numerical variables were conducted as continuous parameters.

SPSS Statistics 22.0 (IBM Corp, Armonk, NY, USA) and R. version 3.4.2 (R foundation for Statistical Computing, Vienna, Austria) were used for statistical analysis. Values with a normal distribution were reported as mean \pm standard deviation (SD) and skewed data were expressed as median and $25-75 \%$ interquartile range (IQR). All statistical tests were two-sided. A $p<0.05$ was considered statistically significant.

\section{Results}

\section{Patient characteristics}

From all 458 patients underwent PD, 288 patients with available preoperative CT were included in the present study. Twenty-three patients were excluded due to preoperative organ failure or incomplete medical data. Finally, 265 patients (136 males and 129 females) were analyzed (Fig. 1). The mean age and BMI were $59.5 \pm 13.9$ years and $23.1 \pm 3.6 \mathrm{~kg} / \mathrm{m}^{2}$, respectively (Table 1). Systemic comorbidities, including hypertension, diabetes, were recorded in 107 (40.4\%) patients. The top three etiologies for PD in our cohort patients were PDAC $(125,47.1 \%)$, ampullar tumor $(78,29.4 \%)$ and pancreatic cystic tumor (34, $12.8 \%)$. Postoperative management was based on the actual patient conditions. According to current nutritional consensus [19], enteral nutrition was considered firstly, PN was initiated if EN was not able to reach $70 \%$ of required calories. Therefore, the average initial parenteral nutrition time (IPNT) was $70.4 \pm$ 13.7 hours after surgery. The average protein delivery (APD) was $1.12 \pm 0.32 \mathrm{~g} / \mathrm{kg} / \mathrm{d}$ calculated during seven days postoperatively. 
Table 1

Basic Characteristics

\begin{tabular}{|c|c|c|c|c|}
\hline & $\begin{array}{l}\text { Low SMI group } \\
(\mathrm{n}=108) \\
\text { Mean/median/n } \\
\text { (SD/IQR/\%) }\end{array}$ & $\begin{array}{l}\text { Normal SMI group } \\
(n=157) \\
\text { Mean/median/n } \\
\text { (SD/IQR/\%) }\end{array}$ & $\begin{array}{l}\text { All Patients } \\
(n=265) \\
\text { Mean/median/n } \\
(\text { SD/IQR/\%) }\end{array}$ & $\mathrm{p}$ \\
\hline Male & $52(48.1 \%)$ & $84(53.5 \%)$ & $136(51.3 \%)$ & 0.212 \\
\hline Age (years) & $62.7 \pm 13.4$ & $55.3 \pm 15.3$ & $59.5 \pm 13.9$ & 0.038 \\
\hline $\mathrm{BMI}\left(\mathrm{kg} / \mathrm{m}^{2}\right)$ & $22.5 \pm 4.1$ & $23.2 \pm 3.9$ & $23.1 \pm 3.6$ & 0.543 \\
\hline NRS2002 & $3.4 \pm 2.3$ & $2.5 \pm 2.1$ & $2.8 \pm 2.5$ & 0.071 \\
\hline $\begin{array}{l}\text { Systemic } \\
\text { comorbidity }\end{array}$ & $45(41.6 \%)$ & $62(39.5 \%)$ & $107(40.4 \%)$ & 0.389 \\
\hline hypertension & $36(33.3 \%)$ & $51(32.5 \%)$ & $87(32.8 \%)$ & 0.412 \\
\hline $\begin{array}{l}\text { Diabetes } \\
\text { mellitus }\end{array}$ & $21(19.4 \%)$ & $25(15.9 \%)$ & $46(17.4 \%)$ & 0.162 \\
\hline Others & $17(15.7 \%)$ & $26(16.6 \%)$ & $43(16.2 \%)$ & 0.321 \\
\hline $\mathrm{SMA}\left(\mathrm{cm}^{2}\right)$ & $118.6 \pm 31.1$ & $155.3 \pm 28.2$ & $138.2 \pm 32.4$ & $\hat{0.001}$ \\
\hline $\mathrm{SMI}\left(\mathrm{cm}^{2} / \mathrm{m}^{2}\right)$ & $38.6 \pm 9.6$ & $49.2 \pm 11.3$ & $46.2 \pm 12.1$ & $\hat{0} .001$ \\
\hline SMD (HU) & $31.4 \pm 8.7$ & $40.6 \pm 9.2$ & $37.2 \pm 10.4$ & $\begin{array}{l}<.001 \\
0.00\end{array}$ \\
\hline SAT $\left(\mathrm{cm}^{2}\right)$ & $127 \pm 26.4$ & $131 \pm 27.3$ & $130 \pm 28.3$ & 0.432 \\
\hline $\operatorname{VAT}\left(\mathrm{cm}^{2}\right)$ & $92.5,43.2-189.1$ & $96.3,41.2-172.3$ & $94.6,42.5-190.6$ & 0.343 \\
\hline $\operatorname{IMAT}\left(\mathrm{cm}^{2}\right)$ & $15.5,8.4-21.4$ & $13.4,9.4-22.5$ & $14.1,8.9-22.1$ & 0.124 \\
\hline \multicolumn{5}{|l|}{ Etiology: } \\
\hline PDAC & 48 (44.4\%) & 77 (49.0\%) & $125(47.1 \%)$ & 0.124 \\
\hline $\begin{array}{l}\text { Pancreatic } \\
\text { cystic tumor }\end{array}$ & $14(13.0 \%)$ & 20 (12.7\%) & $34(12.8 \%)$ & 0.832 \\
\hline pNET & $6(5.6 \%)$ & $9(5.7 \%)$ & $15(5.7 \%)$ & 0.785 \\
\hline
\end{tabular}

SMA: skeletal muscle area, SMI: skeletal muscle index, SMD: skeletal muscle density, SAT:

subcutaneous adipose tissue, VAT: Visceral adipose tissue, IMAT: intermuscular adipose tissue, PDAC: pancreatic ductal adenocarcinoma, pNET: pancreatic neuroendocrine tumors, IPNT: initial parenteral nutrition time, ACD: average calorie delivery, APD: average protein delivery. 


\begin{tabular}{|c|c|c|c|c|}
\hline & $\begin{array}{l}\text { Low SMI group } \\
(\mathrm{n}=108) \\
\text { Mean/median/n } \\
(\mathrm{SD} / \mathrm{QQR} / \%)\end{array}$ & $\begin{array}{l}\text { Normal SMI group } \\
\text { (n=157) } \\
\text { Mean/median/n } \\
\text { (SD/IQR/\%) }\end{array}$ & $\begin{array}{l}\text { All Patients } \\
(\mathrm{n}=265) \\
\text { Mean/median/n } \\
(\mathrm{SD} / \mathrm{QQR} / \%)\end{array}$ & p \\
\hline $\begin{array}{l}\text { Ampullar } \\
\text { tumor }\end{array}$ & 35 (32.4\%) & $43(27.4)$ & 78 (29.4\%) & 0.074 \\
\hline Trauma & $1(0.9 \%)$ & 3 (1.9\%) & $4(1.5 \%)$ & 0.041 \\
\hline Others & $4(3.7 \%)$ & 5 (3.2\%) & 9 (3.4\%) & 0.241 \\
\hline IPNT & $71.3 \pm 12.4$ & $68.3 \pm 15.3$ & $70.4 \pm 13.7$ & 0.432 \\
\hline ACD & $788 \pm 235$ & $764 \pm 265$ & $773 \pm 286$ & 0.543 \\
\hline APD & $1.16 \pm 0.29$ & $1.05 \pm 0.23$ & $1.12 \pm 0.32$ & 0.134 \\
\hline \multicolumn{5}{|c|}{$\begin{array}{l}\text { SMA: skeletal muscle area, SMI: skeletal muscle index, SMD: skeletal muscle density, SAT: } \\
\text { subcutaneous adipose tissue, VAT: Visceral adipose tissue, IMAT: intermuscular adipose tissue, } \\
\text { PDAC: pancreatic ductal adenocarcinoma, pNET: pancreatic neuroendocrine tumors, IPNT: initial } \\
\text { parenteral nutrition time, ACD: average calorie delivery, APD: average protein delivery. }\end{array}$} \\
\hline
\end{tabular}

\section{Body composition analysis}

A sex-specific diagnostic analysis for each CT-derived body composition on major complication (CDC $\geq$ 3) through ROC curve was performed to stratify patient with different risks (Table 2). Among these parameters, SMI has shown the best capability to predict patient developing major complication. The cutoff value of SMI was $47.32 \mathrm{~cm}^{2} / \mathrm{m}^{2}$ for male and $40.65 \mathrm{~cm}^{2} / \mathrm{m}^{2}$ for female, with AUC 0.727 and 0.789 , respectively. Therefore, we divided all the patients to low SMI group and normal SMI group (Table 1). The overall rate of low SMI was $40.75 \%$ (108/265). The two groups showed no difference in sex, BMI, NRS2002 and systemic comorbidity. Both skeletal muscle area (SMA) and skeletal muscle density (SMD) were found significantly different in two groups $\left(118.6 \pm 31.1 \mathrm{~cm}^{2}\right.$ vs. $155.3 \pm 28.2 \mathrm{~cm}^{2}, p<0.001 ; 31.4 \pm$ $8.7 \mathrm{HU}$ vs. $40.6 \pm 9.2 \mathrm{HU}, p<0.001)$. The Subcutaneous adipose tissue (SAT), visceral adipose tissue (VAT) and IMAT were all comparable in the two groups. There was no difference in the IPNT, ACD or APD between the two groups. 
Table 2

Means and sex-specific cut-off values for all CT-scan measurements at L3 level

\begin{tabular}{|c|c|c|c|c|c|c|}
\hline & \multicolumn{4}{|c|}{ Male } & \multicolumn{2}{|l|}{ Female } \\
\hline & $\begin{array}{l}\text { Mean /median } \\
(\mathrm{SD} / \mathrm{IQR})\end{array}$ & Cut-off & AUC & $\begin{array}{l}\text { Mean /median } \\
(\mathrm{SD} / \mathrm{IQR})\end{array}$ & Cut-off & AUC \\
\hline $\mathrm{SMA}\left(\mathrm{cm}^{2}\right)$ & $147.12 \pm 15.21$ & 126.45 & 0.597 & $132.23 \pm 18.62$ & 114.72 & 0.621 \\
\hline $\mathrm{SMI}\left(\mathrm{cm}^{2} / \mathrm{m}^{2}\right)$ & $51.23 \pm 8.21$ & 47.32 & 0.727 & $42.72 \pm 9.56$ & 40.65 & 0.789 \\
\hline SMD (HU) & $38.56 \pm 8.56$ & 36.38 & 0.651 & $32.54 \pm 7.89$ & 31.07 & 0.631 \\
\hline SATI $\left(\mathrm{cm}^{2} / \mathrm{m}^{2}\right)$ & $43.53 \pm 21.12$ & 40.32 & 0.532 & $51.76 \pm 19.42$ & 45.75 & 0.587 \\
\hline $\operatorname{VATI}\left(\mathrm{cm}^{2} / \mathrm{m}^{2}\right)$ & $53.24,29.4-72.4$ & 48.65 & 0.589 & $41.54,27.4-69.3$ & 39.45 & 0.523 \\
\hline IMAT $\left(\mathrm{cm}^{2}\right)$ & $13.34,7.4-18.5$ & 12.18 & 0.612 & $14.22,8.2-20.3$ & 13.76 & 0.593 \\
\hline $\begin{array}{l}\text { SMA: skeletal m } \\
\text { subcutaneous } \\
\text { tissue. }\end{array}$ & $\begin{array}{l}\text { area, SMI: skel } \\
\text { e tissue index, }\end{array}$ & uscl & $x, S$ & $\begin{array}{l}\text { keletal muscle d } \\
\text { sue index, IMAT: }\end{array}$ & $\begin{array}{l}\text { SAT } \\
\text { Iuscu }\end{array}$ & pose \\
\hline
\end{tabular}

\section{The association between skeletal muscle and complications}

The detailed complications and secondary outcome were listed in Table 3, which was grouped by SMI and NRS2002. Eighty-one patients (30.6\%) developed major complication (CDC $\geq 3$ ). POPF and nosocomial infection were observed in $70(26.4 \%)$ and $66(24.9 \%)$ subjects, respectively. The average time span from surgery to discharge was $16.3 \pm 7.2$ days. The overall 28 -day mortality was $1.13 \%(n=3)$. 
Table 3

Primary and secondary outcome measures.

\begin{tabular}{|c|c|c|c|c|c|c|}
\hline & $\begin{array}{l}\text { Low SMI } \\
(n=108)\end{array}$ & $\begin{array}{l}\text { Normal SMI } \\
(n=157)\end{array}$ & $\mathbf{p}$ & $\begin{array}{l}\text { NRS2002 } \leq \\
3(n=194)\end{array}$ & $\begin{array}{l}\text { NRS2002 } \\
\text { ख3 }(n=71)\end{array}$ & $\mathbf{p}$ \\
\hline $\begin{array}{l}\text { Overall major } \\
\text { complication } \\
(n, \%)\end{array}$ & $48(44.4 \%)$ & $33(21.0 \%)$ & $\hat{0} .001$ & $59(30.4 \%)$ & 22(31.0\%) & 0.389 \\
\hline POPF & $39(36.1 \%)$ & $31(19.7 \%)$ & 0.023 & $51(26.3 \%)$ & $19(26.8)$ & 0.637 \\
\hline Biliary leak & $17(15.7 \%)$ & $21(13.4)$ & 0.132 & $27(13.9 \%)$ & $11(15.4 \%)$ & 0.297 \\
\hline Hemorrhage & $12(11.1 \%)$ & $16(10.2 \%)$ & 0.327 & $20(10.3 \%)$ & $8(11.3 \%)$ & 0.193 \\
\hline $\begin{array}{l}\text { Delayed gastric } \\
\text { empting }\end{array}$ & $10(9.2 \%)$ & $16(10.2 \%)$ & 0.041 & $14(7.2 \%)$ & $12(16.9 \%)$ & 0.039 \\
\hline $\begin{array}{l}\text { Nosocomial } \\
\text { infection }\end{array}$ & $37(34.2 \%)$ & $29(18.4 \%)$ & 0.032 & $48(24.7 \%)$ & $18(25.4)$ & 0.412 \\
\hline Cardiac events & $7(6.5 \%)$ & $9(5.7 \%)$ & 0.131 & $11(5.7 \%)$ & $5(7.0 \%)$ & 0.521 \\
\hline Pulmonary events & $28(25.9 \%)$ & $21(13.4 \%)$ & $\hat{0} .001$ & $26(13.4 \%)$ & $23(32.4 \%)$ & 0.021 \\
\hline Renal dysfunction & $11(10.2 \%)$ & $19(12.1 \%)$ & 0.071 & $21(10.8 \%)$ & $9(12.7 \%)$ & 0.721 \\
\hline $\begin{array}{l}\text { Length of } \\
\text { hospitalization (d) }\end{array}$ & $24.5 \pm 6.3$ & $20.4 \pm 5.1$ & 0.123 & $23.1 \pm 5.2$ & $22.8 \pm 6.1$ & 0.439 \\
\hline $\begin{array}{l}\text { Postoperative time } \\
\text { to discharge (d) }\end{array}$ & $18.3 \pm 6.2$ & $15.2 \pm 4.3$ & 0.042 & $16.7 \pm 5.7$ & $17.3 \pm 5.3$ & 0.758 \\
\hline 28-day mortality & $1(0.92 \%)$ & $2(1.27 \%)$ & 0.432 & $2(1.03 \%)$ & $1(1.41 \%)$ & 0.317 \\
\hline
\end{tabular}

At univariable analysis, age, sex, systemic comorbidity, SMI, SMD, and VFA/SMA ratio were significantly associated with major complications (Table 4). Age (OR 1.73, 95\% Cl 1.42-2.15, $\mathrm{p}=0.013)$, systemic comorbidity (OR 1.59, 95\% Cl 1.24-1.92, $\mathrm{p}=0.021$ ) and VFA/SMA (OR $1.23,95 \% \mathrm{Cl} 1.07-1.56, \mathrm{p}=0.012$ ) appeared to be risk factors. Patients with higher SMI (OR 0.72, 95\% Cl 0.54-0.89, p = 0.006) and SMD (OR $0.81,95 \% \mathrm{Cl} 0.72-0.91, p=0.011$ ) tended to have less major complications. Preoperative albumin level, $\mathrm{BMI}$ and the nutritional therapy (including ACD, APD, and IPNT) did not show significant association with major complications. 
Table 4

\Univariable analysis for major complications

\begin{tabular}{|c|c|c|c|c|c|}
\hline \multirow[t]{2}{*}{ Factors } & \multirow{2}{*}{$\begin{array}{l}\mathrm{CDC}<3(\mathrm{n}=184) \\
\text { Mean/median/n } \\
(\mathrm{SD} / \mathrm{IQR} / \%)\end{array}$} & \multirow{2}{*}{$\begin{array}{l}\mathrm{CDC} \geq 3(\mathrm{n}=\mathbf{8 1}) \\
\text { Mean/median/n } \\
(\mathrm{SD} / \mathrm{QQR} / \%)\end{array}$} & \multirow[t]{2}{*}{ OR } & \multirow[t]{2}{*}{$95 \% \mathrm{Cl}$} & \multirow[t]{2}{*}{ p } \\
\hline & & & & & \\
\hline Age (y) & $57.2 \pm 6.7$ & $63.4 \pm 9.5$ & 1.73 & $\begin{array}{l}1.42- \\
2.15\end{array}$ & 0.013 \\
\hline Male & $99,53.8 \%$ & $37,45.7 \%$ & 0.87 & $\begin{array}{l}0.77- \\
0.94\end{array}$ & 0.024 \\
\hline BMI $\left(\mathrm{kg} / \mathrm{m}^{2}\right)$ & $23.5 \pm 4.1$ & $22.7 \pm 5.4$ & 1.09 & $\begin{array}{l}0.95- \\
1.23\end{array}$ & 0.256 \\
\hline NRS2002 & $3.1 \pm 1.7$ & $3.5 \pm 2.1$ & 1.05 & $\begin{array}{l}0.91- \\
1.17\end{array}$ & 0.321 \\
\hline Albumin $(\mathrm{g} / \mathrm{L})$ & $39.1 \pm 5.3$ & $39.4 \pm 6.5$ & 0.98 & $\begin{array}{l}0.94- \\
1.03\end{array}$ & 0.452 \\
\hline $\begin{array}{l}\text { Systemic } \\
\text { comorbidity }\end{array}$ & $58,31.5 \%$ & $43,53.2 \%$ & 1.59 & $\begin{array}{l}1.24- \\
1.92\end{array}$ & 0.021 \\
\hline $\mathrm{SMA}(\mathrm{cm} 2 / \mathrm{m} 2)$ & $148.2 \pm 17.2$ & $131.7 \pm 15.8$ & 0.83 & $\begin{array}{l}0.67- \\
0.94\end{array}$ & 0.023 \\
\hline $\mathrm{SMI}\left(\mathrm{cm}^{2} / \mathrm{m}^{2}\right)$ & $48.3 \pm 13.2$ & $42.6 \pm 14.1$ & 0.72 & $\begin{array}{l}0.54- \\
0.89\end{array}$ & 0.006 \\
\hline SMD (HU) & $39.4 \pm 9.3$ & $35.3 \pm 10.5$ & 0.81 & $\begin{array}{l}0.72- \\
0.91\end{array}$ & 0.011 \\
\hline $\operatorname{SATI}\left(\mathrm{cm}^{2} / \mathrm{m}^{2}\right)$ & $49.14 \pm 18.1$ & $48.37 \pm 23.35$ & 0.97 & $\begin{array}{l}0.91- \\
1.13\end{array}$ & 0.463 \\
\hline VATI $\left(\mathrm{cm}^{2} / \mathrm{m}^{2}\right)$ & $47.33,28.42-69.23$ & $51.26,31.21-73.13$ & 1.11 & $\begin{array}{l}0.83- \\
1.75\end{array}$ & 0.068 \\
\hline IMAT $\left(\mathrm{cm}^{2}\right)$ & $13.21,8.32-18.75$ & $13.78,7.34-21.46$ & 1.04 & $\begin{array}{l}0.92- \\
1.18\end{array}$ & 0.731 \\
\hline VATA/SMA & $1.31,1.07-2.01$ & $1.52,1.23-1.97$ & 1.23 & $\begin{array}{l}1.07- \\
1.56\end{array}$ & 0.012 \\
\hline IPNT (h) & $69.3 \pm 12.7$ & $71.2 \pm 14.5$ & 1.14 & $\begin{array}{l}0.92- \\
1.32\end{array}$ & $\begin{array}{l}0 . \\
322\end{array}$ \\
\hline $\mathrm{ACD}(\mathrm{kcal} / \mathrm{kg} / \mathrm{d})$ & $15.8 \pm 3.9$ & $16.4 \pm 4.42$ & 1.03 & $\begin{array}{l}0.87- \\
1.27\end{array}$ & 0.156 \\
\hline $\operatorname{APD}(\mathrm{g} / \mathrm{kg} / \mathrm{d})$ & $1.15 \pm 0.21$ & $1.08 \pm 0.32$ & 0.91 & $\begin{array}{l}0.79- \\
1.23\end{array}$ & 0.121 \\
\hline
\end{tabular}


Multivariable analysis was constructed by considering the body composition parameters as dichotomous variables, based on the previously calculated cut-points. Multivariable analysis showed age, SMI and SMD were independent predictors for major complications, with ORs of 1.49 (95\% Cl 1.22-1.83, $p=$ $0.026), 0.77(95 \% \mathrm{Cl} 0.51-0.94, \mathrm{p}=0.015)$ and $0.85(95 \% \mathrm{Cl} 0.64-1.03, \mathrm{p}=0.029)$. However, sex, systemic comorbidity and VATA/SMA did not show significant effect (Table 5). Based on this multivariable model, we tested its predictive performance on major complication. ROC curve shown the AUC was 0.776 , with a sensitivity of $63.3 \%$ and a specificity of $83.3 \%$ in its optimal cut-point (Fig. 2).

Table 5

Estimated effects of the parameters on major complications in multivariable logistic regression.

\begin{tabular}{|llll|}
\hline Factor & OR & $95 \%$ Cl & p \\
\hline Sex & 0.98 & $0.87-1.18$ & 0.259 \\
\hline Age & 1.49 & $1.22-1.83$ & 0.026 \\
SMI & 0.77 & $0.51-0.94$ & 0.015 \\
SMD & 0.85 & $0.64-1.03$ & 0.029 \\
VATA/SMA & 1.12 & $0.79-1.35$ & 0.351 \\
Systemic morbidity & 1.23 & $0.82-1.42$ & 0.189 \\
\hline $\begin{array}{l}\text { SMI: skeletal muscle index, SMD: skeletal muscle density, VATA/SMA: visceral adipose tissue } \\
\text { area/skeletal muscle area }\end{array}$ & & \\
\hline
\end{tabular}

\section{Subgroup analysis in sarcopenic patients}

Additional subgroup analysis was conducted in low and normal SMI patients respectively. (Table 6). Multivariable logistic regression showed, besides age (OR 2.21, 95\% 1.73-2.84, $p=0.003$ ) and VATI (OR1.45, 95\% Cl 1.19-2.04, p = 0.017), IPNT (OR 1.89, 95\% Cl 1.43-2.49, p = 0.032) and APD (OR 0.76, $95 \% \mathrm{Cl} 0.53-0.89, \mathrm{p}=0.021$ ) were also significantly associated with major complications in low SMI group. However, this result did not happen in patients with normal SMI. Furthermore, multivariable adjusted restricted cubic splines (RCS) for the association between IPNT, APD and major complications were shown in Fig. 3, with the mean values as the reference standard. 
Table 6

Multivariable logistic regression analysis in low SMI and normal SMI group

\begin{tabular}{|c|c|c|c|c|c|c|}
\hline & \multicolumn{3}{|c|}{ low SMI } & \multicolumn{3}{|c|}{ normal SMI } \\
\hline & OR & $95 \% \mathrm{Cl}$ & $\mathrm{p}$ & OR & $95 \% \mathrm{Cl}$ & $\mathrm{p}$ \\
\hline Age & 2.21 & $1.73-2.84$ & 0.003 & 1.19 & $1.01-1.31$ & 0.039 \\
\hline Systemic comorbidity & 1.19 & $0.65-1.87$ & 0.129 & 1.07 & $0.83-1.35$ & 0.237 \\
\hline VATI & 1.45 & $1.19-2.04$ & 0.017 & 1.13 & $0.81-1.53$ & 0.098 \\
\hline IPNT & 1.89 & $1.43-2.49$ & 0.032 & 0.96 & $0.79-1.23$ & 0.342 \\
\hline APD & 0.76 & $0.53-0.89$ & 0.021 & 1.04 & $0.83-1.27$ & 0.247 \\
\hline
\end{tabular}

\section{Discussion}

Pancreaticoduodenectomy is one of the major procedures for periampullary malignancy and benign diseases. However, the high rate of postoperative complications hinders the rehabilitation and increases medical cost. Other than improving surgical techniques, identifying susceptible patients and refining support strategy seems crucial. In the present study, we found preoperative skeletal muscle index and density as well as age were prognostic factors affecting postoperative complications. More importantly, through sub-group analysis, the IPNT and APD were independent determinants for complications in patients with lower SMI. These results may provide a practical strategy for distinguishing PD patients of high risk and tailored nutritional therapy.

Previously, Martin et al demonstrated skeletal muscle depletion was a powerful prognostic factor for survival in cancer patients [1]. In critical patients, muscle wasting was prevalent and profound. Up to 60$70 \%$ critical patients had muscle wasting at ICU admission [20], and $20 \%$ of skeletal muscle reduction was found in ICU patients within first 10 days [21]. Both the quantity and quality of skeletal muscle affected the course and outcome of the disease $[2,3]$. In our present study, preoperative muscle depletion was found in $40.75 \%$ subjects who would undergo PD. The reported incidence rate of sarcopenia in PD patients varied a lot from $24.2 \%$ [22] to $75.5 \%$ [7, 23], which may due to different population and cut-off value. Pancreatic and periampullary cancer patients were always malnourished at admission. It was necessary to have a thorough and accurate evaluation on the nutritional status. To date, CT was a widely used method for measuring skeletal muscle conditions due to its accuracy [23], and other approaches included bioelectrical impedance analysis and ultrasound. In our univariable analysis, VATA/SMA ratio appeared to be a significant factor affecting postoperative morbidity (RR 1.23, 95\% Cl 1.07-1.56, P = 0.012). This was in accordance with previous studies. David et al have shown high visceral adipose tissue was associated with increased infection rate (OR: 2.4) in pancreatic cancer patients. Moreover, longer survival was observed in patients with high muscle attenuation combined with low visceral 
adipose tissue $(P=0.011)$ [4]. In cancer patients underwent $P D$, Sandini et al had shown a high visceral adipose tissue-to-skeletal muscle ratio significantly increased major complication with $\mathrm{OR}$ reaching 3.20 [22]. From a prospective study with 284 patients undergoing PD, researchers demonstrated sarcopenic obesity was the only independent predictor for POPF (OR 2.65, 95\% Cl 1.43-4.93) [7]. In addition, females tend to have higher rate of sarcopenic obesity $(\mathrm{BMI}>25)$ than males ( $38 \% \mathrm{vs.} 12 \%)$ in patients with resectable PDAC and those patients showed higher incidence of major complications $(P<0.001)[5]$. Adipose tissue does have some merits, such as energy storage, harm protection, warm keeping. Being a double-edge sword, fat may also play a bad character under some conditions. Excessive visceral fat could handicap the surgical operation, increasing the intraoperative risk. As an endocrine organ, unbalanced adipokines and cytokines may promote postoperative inflammation and develop insulin resistance [24].

In the multivariable regression, we found preoperative L3 level muscle index and density were predictive of postoperative complications. Here, higher muscle density was significantly related reduced complications $(\mathrm{OR}=0.85,95 \% \mathrm{Cl} 0.64-1.03, \mathrm{p}=0.029)$. The result was in line with other studies. Amini et al reported higher risk of morbidity and complication was observed in patients with sarcopenia after curative resection for pancreatic adenocarcinoma [25]. Recently, both Nicolas and Minji et al have demonstrated mean muscle attenuation could be a promising parameter to predict complications and POPF after PD [6, 7]. In addition to short-term results, long-term outcome also seems to relate to skeletal muscle conditions. Elisabeth et al have shown sarcopenia negatively impacted overall survival in resectable PDCA patients (14 vs. 20 months) [5]. However, several studies demonstrated the amount of muscle mass was not predictive of major complication or morbidity after pancreatic surgery [22, 26]. We considered the disagreement may relate to different methods, cut-off values and etiology.

Lower skeletal muscle area and density probably mean lower protein reserve and myosteatosis. In aging and tumor patients, the muscle depletion may be caused by insufficient intake and ensuing proteolysis, which occurred long before admission. Study has shown the serum albumin concentration correlated well with skeletal mass in critical patients [27]. Decreased albumin always indicated poor nutrition status and even leaded to tissue edema, which may hamper surgical procedure and healing. Reduced muscle density was a reflection of alteration in muscle composition within muscle fibers, as fluid surcharge and fat accumulation [28]. In several researches of oncology, including melanoma, adrenocortical carcinoma, renal cell carcinoma, muscle density was found to be an independent prognostic factor of survival [2931]. An inverse association between muscle attenuation and triglyceride content has been established in healthy adults [32], and it was shown IMAT correlated with muscle density [4]. Myosteatosis may involve insulin resistance, inflammation, mitochondrial damage and oxidative stress [27]. These metabolic changes would further cause a fuel utilization shift from lipids to glucose in muscle, leading to muscle protein depletion and reduced capacity [33].

Of particular interesting, our subgroup analysis revealed that delayed initiation of supplementary parental nutrition (SPN) was associated with higher complications in low SMI patients undergoing PD (OR 1.89, $95 \% \mathrm{Cl} 1.43-2.49, \mathrm{p}=0.032$ ). It was widely recognized that early enteral feeding was preferred in major 
surgery and critical patients $[11,12,19]$. However, enteral feeding always accompanied with abdominal distention, nausea, vomiting and underfeeding, especially among patients receiving major upper gastrointestinal surgery. According to ASPEN guideline, PN should be delayed for 5-7 days in the postoperative ICU patients, who may partly represent PD subjects [11]. However, the rationale resources it based on were largely from clinical studies conducted more than twenty years ago [13,34,35], which may differ from current PN pattern including preparation technology and hypocaloric feeding. Actually, in a recent multicenter RCT comparing EN and PN, no difference in mortality, infectious complication rate, and hospital length of stay was observed in critical patients [36]. In malnourished patients, PN resulted in a significantly lower mortality with a tendency towards lower rates of infection [37]. The timing of starting PN has long been debated in critical and surgical patients $[11,12,19,38]$. Our present study, to our knowledge, for the first time demonstrated only in low SMI patients, early PN was beneficial for reduced complication. This result was in line with Heidegger's research, which found in malnourished patients and those with special risks SPN should be considered on day 4 after ICU admission [39]. Indeed, many studies have yielded negative results on early PN support in critical and surgical patients [38, 40]. However, a recent Cochrane meta-analysis could not draw a clear conclusion as whether early or late SPN was better in critical patients due to low-quality evidence [41]. The heterogeneity and different baseline nutrition status may have impacted the results. ESPEN guideline suggested PN should be considered in high nutrition risk or malnourished patients $[12,19]$. But, no clear prescription was provided as a lack of integrating studies. Since skeletal muscle was a crucial indicator for nutrition status and affected patients outcome both in short and long term [2,3,21], we tested whether it could differentiate the efficiency of parental nutrition support in PD patients. The present results may help identify those patients who may benefit early PN support and provide some evidence for early SPN in sarcopenic patients.

Some mechanism researches have shown early PN may suppress muscle cell autophagy and associated with muscle weakness $[42,43]$. In addition, increased amount of adipose tissue within the muscle compartments was observed in critical patients receiving early PN [40]. However, these outcomes did not mean worse endpoints. Perhaps, it may just represent a metabolic process. Anyhow, early nutrition support and sufficient protein supply can ensure necessary substrates in malnourished patients, even though it may not affect muscle wasting.

Here we also have shown higher protein supply was a protective factor for postoperative complication in low SMI patients (OR $0.76,95 \% \mathrm{Cl} 0.53-0.89, \mathrm{p}=0.021)$. This result was supported by previous observational researches $[44,45]$, which demonstrated higher protein delivery was associated with improved survival. Benefits of high protein delivery were also found in RCT trials. Ferrie et al shown higher level of amino acids supply $(1.1 \mathrm{~g} / \mathrm{kg}$ vs $0.9 \mathrm{~g} / \mathrm{kg})$ was associated with improved patients focused outcomes, such as handgrip strength and muscle mass, despite without differences in mortality [46]. Doig et al found higher intravenous amino acid therapy was related to improvement in renal function [47]. Ishibashi et al shown higher protein intake $(>1.5 \mathrm{~g} / \mathrm{kg} / \mathrm{d})$ was associated with less total body protein loss[48]. Mechanistic studies have shown a 3-h high level $(1 \mathrm{~g} / \mathrm{kg} / \mathrm{d})$ amino acid infusion was able to improve protein balance from negative to positive in critical patients [49]. Sundstrom et al also reported a 
supplemental intravenous amino acid infusion sustained a positive protein balance for $24 \mathrm{~h} \mathrm{[50].} \mathrm{Current}$ guidelines recommend adequate protein supply ( $\geq 1.2$ or $1.3 \mathrm{~g} / \mathrm{kg} / \mathrm{d}$ ) in critical patients, but few data exists as to major surgery patients [19]. Therefore, sufficient protein supply appears beneficial in surgical patients, but high quality evidence is needed.

Our study did have some limitations that should be addressed. First, the patient population underwent PD procedure was due to various reasons from trauma, benign disease to cancer. This heterogeneity may reduce the quality of evidence to guide clinical case. Second, we only analyzed the relationship between preoperative skeletal muscle and short-term postoperative complications. The extent of muscle wasting after surgery was not documented, which may also influence the short and long-term outcomes. Thus, dynamic courses of skeletal muscle conditions and its impact on prognosis as well as nutrition support should be examined in the future. Third, the patients who received late PN may have more severe conditions, such as hemodynamic instability, which may add bias to the analysis.

\section{Conclusion}

Low skeletal muscle index and density were independent risk factors for postoperative complications in PD patients. Therefore, preoperative body composition analysis by CT scan seems potential for the stratification of complication risk. For patients with low SMI, early PN initiation and sufficient protein supply appears to be a protective factor for postoperative complications in PD patients, but large RCT trials are required to validate this result.

\section{Abbreviations}

PD: pancreaticoduodenectomy; SMA: skeletal muscle area; SMI: skeletal muscle index; SMD: skeletal muscle density; IMAT: intermuscular adipose tissue; SAT: subcutaneous adipose tissue; SATI: subcutaneous adipose tissue index; VAT: Visceral adipose tissue; VATI: Visceral adipose tissue index; VATA: visceral adipose tissue area; IPNT: initial parenteral nutrition time; APD: average protein delivery; ACD: average calories delivery; ICU: intensive care unit; PDAC: pancreatic ductal adenocarcinoma; POPF: postoperative pancreatic fistula; EN: enteral nutrition; PN: parenteral nutrition; BMI: body mass index; CDC: Clavien-Dindo Classification; HU: Hounsfield Units; ROC: receiver operating characteristic; RCT: randomized control trial.

\section{Declarations}

\section{Acknowledgments}

None.

\section{Funding}


This research was supported by National Natural Science Foundation (81700788).

\section{Availability of data and materials}

The datasets generated and analyzed during the current study are available from the corresponding author on reasonable request.

\section{Author's contributions}

Conceived and designed the study: Dongming Zhu, Jin Zhou. Kaipeng Duan. Data collection and analysis: Kaipeng Duan, Xin Gao, Mengting Gong, Drafting the article: Kaipeng Duan, Luxin Wei. Final approval of the version to be submitted: all authors.

\section{Competing interests}

The authors declare that they have no competing interests. This research was supported by National Natural Science Foundation (81700788).

\section{Consent for publication}

All images in this manuscript are entirely unidentifiable and do not include any personal details, therefore no consent for publication was obtained

\section{Ethics approval and consent to participate}

The study was approved by the Ethics Committee of The First Affiliated Hospital of Soochow University. The need for informed consent was waived because of the retrospective nature of the study using only data obtained from standard care.

\section{References}

1. Martin L, Birdsell L, Macdonald N, Reiman T, Clandinin MT, McCargar LJ, Murphy R, Ghosh S, Sawyer $M B$, Baracos VE. Cancer cachexia in the age of obesity: skeletal muscle depletion is a powerful prognostic factor, independent of body mass index. Journal of clinical oncology: official journal of the American Society of Clinical Oncology. 2013;31(12):1539-47.

2. Weijs PJ, Looijaard WG, Dekker IM, Stapel SN, Girbes AR, Oudemans-van Straaten HM, Beishuizen A. Low skeletal muscle area is a risk factor for mortality in mechanically ventilated critically ill patients. Critical care. 2014;18(2):R12. 
3. Looijaard WG, Dekker IM, Stapel SN, Girbes AR, Twisk JW, Oudemans-van Straaten HM, Weijs PJ. Skeletal muscle quality as assessed by CT-derived skeletal muscle density is associated with 6month mortality in mechanically ventilated critically ill patients. Critical care. 2016;20(1):386.

4. van Dijk DP, Bakens MJ, Coolsen MM, Rensen SS, van Dam RM, Bours MJ, Weijenberg MP, Dejong $\mathrm{CH}$, Olde Damink SW. Low skeletal muscle radiation attenuation and visceral adiposity are associated with overall survival and surgical site infections in patients with pancreatic cancer. Journal of cachexia sarcopenia muscle. 2017;8(2):317-26.

5. Gruber ES, Jomrich G, TamandI D, Gnant M, Schindl M, Sahora K. Sarcopenia and sarcopenic obesity are independent adverse prognostic factors in resectable pancreatic ductal adenocarcinoma. PloS one. 2019;14(5):e0215915.

6. Linder N, Schaudinn A, Langenhan K, Krenzien F, Hau HM, Benzing C, Atanasov G, Schmelzle M, Kahn T, Busse $\mathrm{H}$, et al. Power of computed-tomography-defined sarcopenia for prediction of morbidity after pancreaticoduodenectomy. BMC Med Imaging. 2019;19(1):32.

7. Jang M, Park HW, Huh J, Lee JH, Jeong YK, Nah YW, Park J, Kim KW. Predictive value of sarcopenia and visceral obesity for postoperative pancreatic fistula after pancreaticoduodenectomy analyzed on clinically acquired CT and MRI. European radiology. 2019;29(5):2417-25.

8. Schroder FF, de Graaff F, Bouman DE, Brusse-Keizer M, Slump KH, Klaase JM. The Preoperative CTScan Can Help to Predict Postoperative Complications after Pancreatoduodenectomy. BioMed research international. 2015;2015:824525.

9. Cruz-Jentoft AJ, Bahat G, Bauer J, Boirie Y, Bruyere O, Cederholm T, Cooper C, Landi F, Rolland Y, Sayer AA, et al. Sarcopenia: revised European consensus on definition and diagnosis. Age Ageing. 2019;48(1):16-31.

10. Haldar S, Chia SC, Henry CJ. Body Composition in Asians and Caucasians: Comparative Analyses and Influences on Cardiometabolic Outcomes. Adv Food Nutr Res. 2015;75:97-154.

11. Taylor BE, McClave SA, Martindale RG, Warren MM, Johnson DR, Braunschweig C, McCarthy MS, Davanos E, Rice TW, Cresci GA, et al. Guidelines for the Provision and Assessment of Nutrition Support Therapy in the Adult Critically III Patient: Society of Critical Care Medicine (SCCM) and American Society for Parenteral and Enteral Nutrition (A.S.P.E.N.). Critical care medicine. 2016;44(2):390-438.

12. Singer $P$, Blaser AR, Berger MM, Alhazzani W, Calder PC, Casaer MP, Hiesmayr M, Mayer K, Montejo JC, Pichard C, et al. ESPEN guideline on clinical nutrition in the intensive care unit. Clinical nutrition. 2019;38(1):48-79.

13. Klein S, Kinney J, Jeejeebhoy K, Alpers D, Hellerstein M, Murray M, Twomey P. Nutrition support in clinical practice: review of published data and recommendations for future research directions. National Institutes of Health, American Society for Parenteral and Enteral Nutrition, and American Society for Clinical Nutrition. JPEN Journal of parenteral enteral nutrition. 1997;21(3):133-56.

14. Doig GS, Simpson F, Sweetman EA, Finfer SR, Cooper DJ, Heighes PT, Davies AR, O'Leary M, Solano T, Peake $S$, et al. Early parenteral nutrition in critically ill patients with short-term relative 
contraindications to early enteral nutrition: a randomized controlled trial. Jama. 2013;309(20):21308.

15. Dindo D, Demartines N, Clavien PA. Classification of surgical complications: a new proposal with evaluation in a cohort of 6336 patients and results of a survey. Annals of surgery. 2004;240(2):20513.

16. Bassi C, Dervenis C, Butturini G, Fingerhut A, Yeo C, Izbicki J, Neoptolemos J, Sarr M, Traverso W, Buchler M, et al. Postoperative pancreatic fistula: an international study group (ISGPF) definition. Surgery. 2005;138(1):8-13.

17. Shen W, Punyanitya M, Wang Z, Gallagher D, St-Onge MP, Albu J, Heymsfield SB, Heshka S. Total body skeletal muscle and adipose tissue volumes: estimation from a single abdominal crosssectional image. J Appl Physiol. 2004;97(6):2333-8.

18. Mourtzakis M, Prado CM, Lieffers JR, Reiman T, McCargar LJ, Baracos VE. A practical and precise approach to quantification of body composition in cancer patients using computed tomography images acquired during routine care. Applied physiology, nutrition, and metabolism = Physiologie appliquee, nutrition et metabolisme 2008, 33(5):997-1006.

19. Weimann A, Braga M, Carli F, Higashiguchi T, Hubner M, Klek S, Laviano A, Ljungqvist O, Lobo DN, Martindale R, et al. ESPEN guideline: Clinical nutrition in surgery. Clinical nutrition. 2017;36(3):62350 .

20. Looijaard W, Molinger J, Weijs PJM. Measuring and monitoring lean body mass in critical illness. Curr Opin Crit Care. 2018;24(4):241-7.

21. Puthucheary ZA, Rawal J, McPhail M, Connolly B, Ratnayake G, Chan P, Hopkinson NS, Phadke R, Dew T, Sidhu PS, et al. Acute skeletal muscle wasting in critical illness. Jama. 2013;310(15):1591600.

22. Sandini M, Bernasconi DP, Fior D, Molinelli M, Ippolito D, Nespoli L, Caccialanza R, Gianotti L. A high visceral adipose tissue-to-skeletal muscle ratio as a determinant of major complications after pancreatoduodenectomy for cancer. Nutrition. 2016;32(11-12):1231-7.

23. Nishida Y, Kato Y, Kudo M, Aizawa H, Okubo S, Takahashi D, Nakayama Y, Kitaguchi K, Gotohda N, Takahashi S, et al. Preoperative Sarcopenia Strongly Influences the Risk of Postoperative Pancreatic Fistula Formation After Pancreaticoduodenectomy. Journal of gastrointestinal surgery: official journal of the Society for Surgery of the Alimentary Tract. 2016;20(9):1586-94.

24. Lehr S, Hartwig S, Sell H. Adipokines: a treasure trove for the discovery of biomarkers for metabolic disorders. Proteomics Clinical applications. 2012;6(1-2):91-101.

25. Amini N, Spolverato G, Gupta R, Margonis GA, Kim Y, Wagner D, Rezaee N, Weiss MJ, Wolfgang CL, Makary MM, et al. Impact Total Psoas Volume on Short- and Long-Term Outcomes in Patients Undergoing Curative Resection for Pancreatic Adenocarcinoma: a New Tool to Assess Sarcopenia. Journal of gastrointestinal surgery: official journal of the Society for Surgery of the Alimentary Tract. 2015;19(9):1593-602. 
26. Peng P, Hyder O, Firoozmand A, Kneuertz P, Schulick RD, Huang D, Makary M, Hirose K, Edil B, Choti MA, et al. Impact of sarcopenia on outcomes following resection of pancreatic adenocarcinoma. Journal of gastrointestinal surgery: official journal of the Society for Surgery of the Alimentary Tract. 2012;16(8):1478-86.

27. Dusseaux MM, Antoun S, Grigioni S, Beduneau G, Carpentier D, Girault C, Grange S, Tamion F. Skeletal muscle mass and adipose tissue alteration in critically ill patients. PloS one. 2019;14(6):e0216991.

28. Zhang P, Peterson M, Su GL, Wang SC. Visceral adiposity is negatively associated with bone density and muscle attenuation. Am J Clin Nutr. 2015;101(2):337-43.

29. Sabel MS, Lee J, Cai S, Englesbe MJ, Holcombe S, Wang S. Sarcopenia as a prognostic factor among patients with stage III melanoma. Ann Surg Oncol. 2011;18(13):3579-85.

30. Miller BS, Ignatoski KM, Daignault S, Lindland C, Doherty M, Gauger PG, Hammer GD, Wang SC, Doherty GM. University of Michigan Analytical Morphomics G: Worsening central sarcopenia and increasing intra-abdominal fat correlate with decreased survival in patients with adrenocortical carcinoma. World journal of surgery. 2012;36(7):1509-16.

31. Antoun S, Lanoy E, lacovelli R, Albiges-Sauvin L, Loriot Y, Merad-Taoufik M, Fizazi K, di Palma M, Baracos VE, Escudier B. Skeletal muscle density predicts prognosis in patients with metastatic renal cell carcinoma treated with targeted therapies. Cancer. 2013;119(18):3377-84.

32. Goodpaster BH, Kelley DE, Thaete FL, He J, Ross R. Skeletal muscle attenuation determined by computed tomography is associated with skeletal muscle lipid content. J Appl Physiol. 2000;89(1):104-10.

33. Taaffe DR, Henwood TR, Nalls MA, Walker DG, Lang TF, Harris TB. Alterations in muscle attenuation following detraining and retraining in resistance-trained older adults. Gerontology. 2009;55(2):21723.

34. Brennan MF, Pisters PW, Posner M, Quesada O, Shike M. A prospective randomized trial of total parenteral nutrition after major pancreatic resection for malignancy. Annals of surgery. 1994;220(4):436-41. discussion 441 - 434.

35. Sandstrom R, Drott C, Hyltander A, Arfvidsson B, Schersten T, Wickstrom I, Lundholm K. The effect of postoperative intravenous feeding (TPN) on outcome following major surgery evaluated in a randomized study. Annals of surgery. 1993;217(2):185-95.

36. Harvey SE, Parrott F, Harrison DA, Bear DE, Segaran E, Beale R, Bellingan G, Leonard R, Mythen MG, Rowan KM, et al. Trial of the route of early nutritional support in critically ill adults. N Engl J Med. 2014;371(18):1673-84.

37. Braunschweig CL, Levy P, Sheean PM, Wang X. Enteral compared with parenteral nutrition: a metaanalysis. Am J Clin Nutr. 2001;74(4):534-42.

38. Casaer MP, Mesotten D, Hermans G, Wouters PJ, Schetz M, Meyfroidt G, Van Cromphaut S, Ingels C, Meersseman P, Muller J, et al. Early versus late parenteral nutrition in critically ill adults. $\mathrm{N}$ Engl J Med. 2011;365(6):506-17. 
39. Heidegger CP, Berger MM, Graf S, Zingg W, Darmon P, Costanza MC, Thibault R, Pichard C. Optimisation of energy provision with supplemental parenteral nutrition in critically ill patients: a randomised controlled clinical trial. Lancet. 2013;381(9864):385-93.

40. Casaer MP, Langouche L, Coudyzer W, Vanbeckevoort D, De Dobbelaer B, Guiza FG, Wouters PJ, Mesotten D, Van den Berghe G. Impact of early parenteral nutrition on muscle and adipose tissue compartments during critical illness. Critical care medicine. 2013;41(10):2298-309.

41. Fuentes Padilla P, Martinez G, Vernooij RW, Urrutia G, Roque IFM, Bonfill Cosp X. Early enteral nutrition (within $\mathbf{4 8}$ hours) versus delayed enteral nutrition (after 48 hours) with or without supplemental parenteral nutrition in critically ill adults. The Cochrane database of systematic reviews 2019, 2019(10).

42. Hermans G, Casaer MP, Clerckx B, Guiza F, Vanhullebusch T, Derde S, Meersseman P, Derese I, Mesotten D, Wouters PJ, et al. Effect of tolerating macronutrient deficit on the development of intensive-care unit acquired weakness: a subanalysis of the EPaNIC trial. The Lancet Respiratory medicine. 2013;1(8):621-9.

43. Derde S, Vanhorebeek I, Guiza F, Derese I, Gunst J, Fahrenkrog B, Martinet W, Vervenne H, Ververs EJ, Larsson L, et al. Early parenteral nutrition evokes a phenotype of autophagy deficiency in liver and skeletal muscle of critically ill rabbits. Endocrinology. 2012;153(5):2267-76.

44. Weijs PJ, Looijaard WG, Beishuizen A, Girbes AR, Oudemans-van Straaten HM. Early high protein intake is associated with low mortality and energy overfeeding with high mortality in non-septic mechanically ventilated critically ill patients. Critical care. 2014;18(6):701.

45. Nicolo M, Heyland DK, Chittams J, Sammarco T, Compher C. Clinical Outcomes Related to Protein Delivery in a Critically III Population: A Multicenter, Multinational Observation Study. JPEN Journal of parenteral enteral nutrition. 2016;40(1):45-51.

46. Ferrie S, Allman-Farinelli M, Daley M, Smith K. Protein Requirements in the Critically III: A Randomized Controlled Trial Using Parenteral Nutrition. JPEN Journal of parenteral enteral nutrition. 2016;40(6):795-805.

47. Doig GS, Simpson F, Bellomo R, Heighes PT, Sweetman EA, Chesher D, Pollock C, Davies A, Botha J, Harrigan P, et al. Intravenous amino acid therapy for kidney function in critically ill patients: a randomized controlled trial. Intensive care medicine. 2015;41(7):1197-208.

48. Ishibashi N, Plank LD, Sando K, Hill GL. Optimal protein requirements during the first 2 weeks after the onset of critical illness. Critical care medicine. 1998;26(9):1529-35.

49. Liebau F, Sundstrom M, van Loon LJ, Wernerman J, Rooyackers $O$. Short-term amino acid infusion improves protein balance in critically ill patients. Critical care. 2015;19:106.

50. Sundstrom Rehal M, Liebau F, Tjader I, Norberg A, Rooyackers O, Wernerman J. A supplemental intravenous amino acid infusion sustains a positive protein balance for 24 hours in critically ill patients. Critical care. 2017;21(1):298.

\section{Figures}




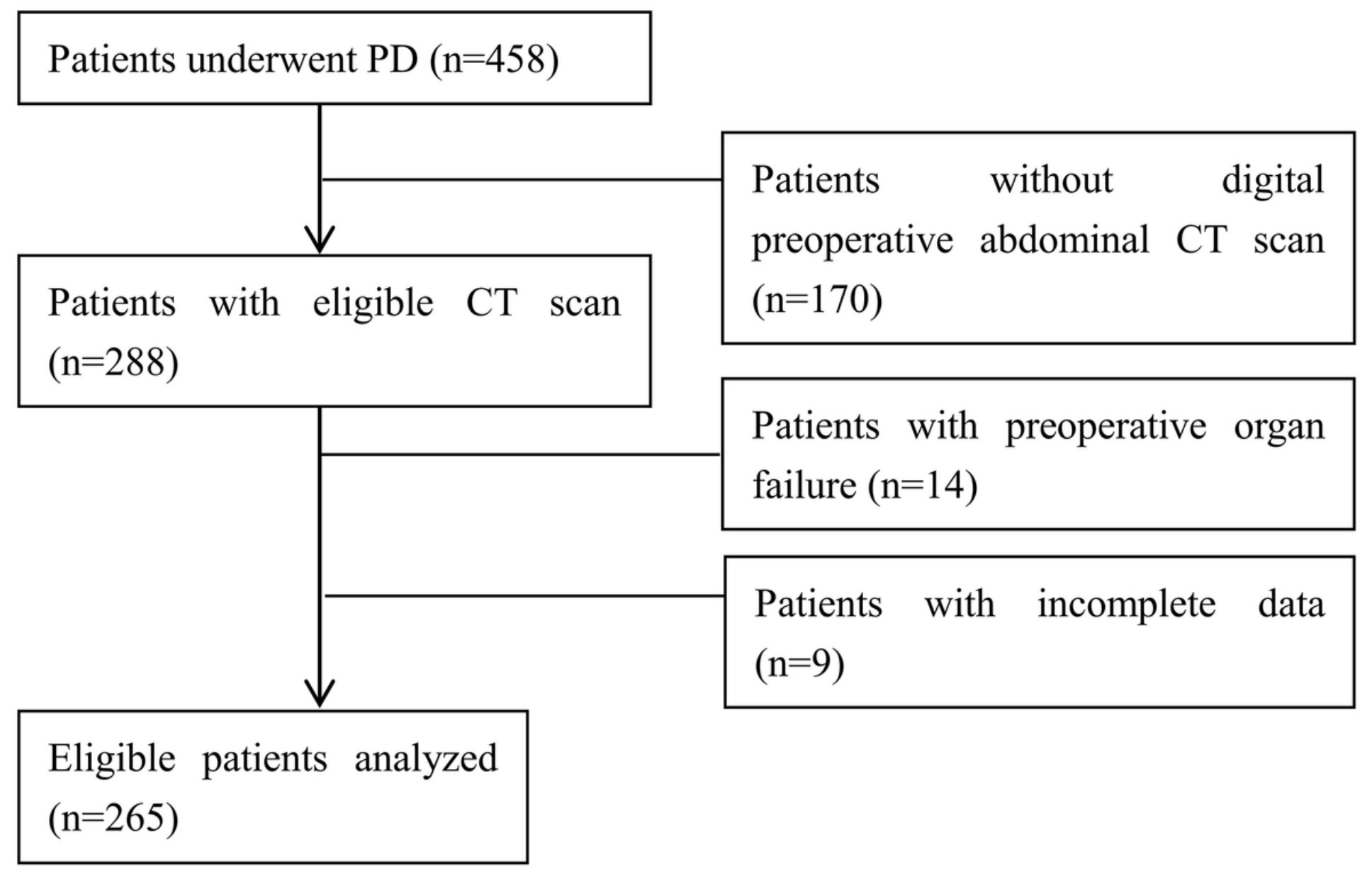

Figure 1

Flowchart of patients included into the analysis 


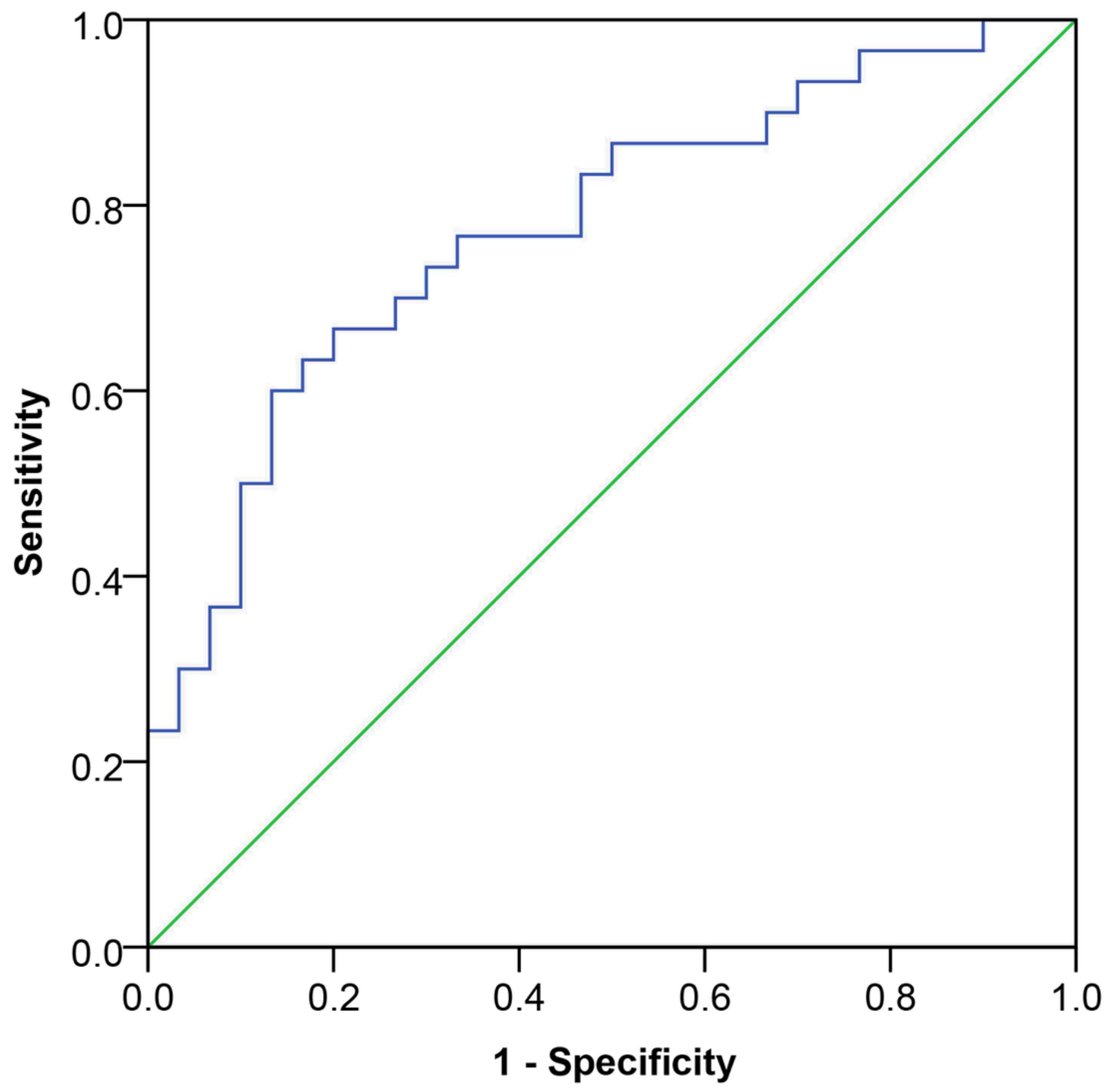

Figure 2

ROC curve for the selected logistic regression model in the diagnosis of postoperative complication. Area under the curve $=0.776$; sensitivity $=63.3 \%$; specificity $=83.3 \%$. 

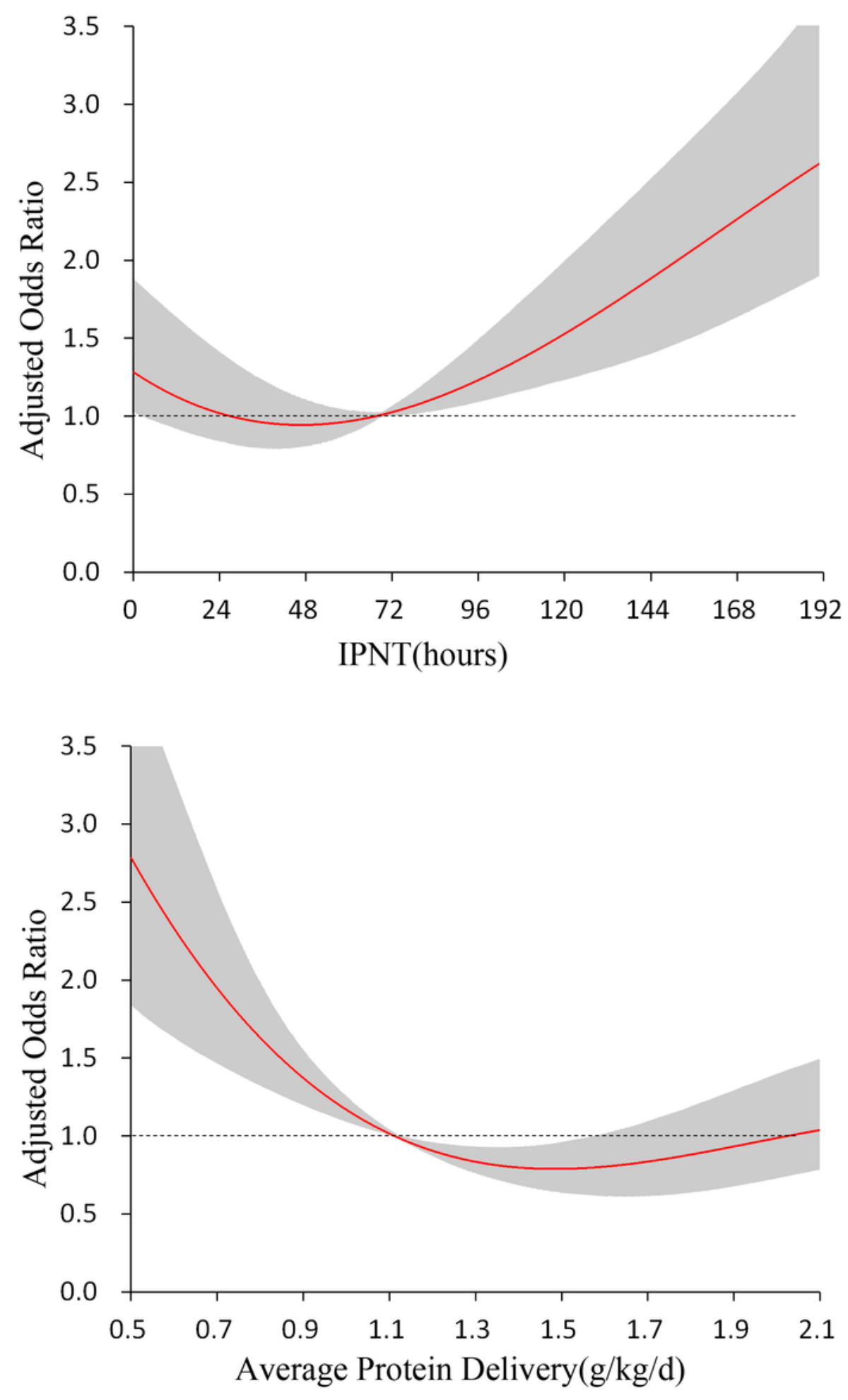

\section{Figure 3}

Association between IPNT (a), APD (b) and postoperative complication in subgroup patients with low SMI. Data were fit by a multivariable logistic regression model based on restricted cubic splines. The mean IPTN and APD were defined as the reference standard. The grey area represents the $95 \% \mathrm{Cl}$. 